\title{
Chemical Vapour Deposition of Hexagonal Boron Nitride for Two Dimensional Electronics
}

\begin{abstract}
Balaji Sompalle ${ }^{1}$, Jérôme Borme ${ }^{2}$, Fátima Cerqueira ${ }^{3}$, Tangyou Sun ${ }^{4}$, Rui Campos ${ }^{5}$, Pedro Alpuim 6

${ }^{1}$ Center of Physics, University of Minho, Braga, Portugal; 2D Materials and Devices Research Group, International Iberian Nanotechnology laboratory, Braga, Portugal (sgds_balaji@yahoo.com) ORCID 0000-0001-6866-1137; 22D Materials and Devices Research Group, International Iberian Nanotechnology laboratory, Braga, Portugal (jerome.borme@inl.int) ORCID 0000-0002-5622-7760; ${ }^{3}$ Center of Physics, University of Minho, Braga, Portugal; 2D Materials and Devices Research Group, International Iberian Nanotechnology laboratory, Braga, Portugal (fatima.cerqueira.vt@inl.int) ORCID 0000-00023505-6982; ' Center of Physics, University of Minho, Braga, Portugal; 2D Materials and Devices Research Group, International Iberian Nanotechnology laboratory, Braga, Portugal (tangyou.sun@inl.int) ORCID 0000-0002-5885-985X; ${ }^{5}$ Center of Physics, University of Minho, Braga, Portugal; 2D Materials and Devices Research Group, International Iberian Nanotechnology laboratory, Braga, Portugal (rui.campos@inl.int) ORCID 0000-0002-84582356; ${ }^{6}$ Center of Physics, University of Minho, Braga, Portugal; 2D Materials and Devices Research Group, International Iberian Nanotechnology laboratory, Braga, Portugal (pedro.alpuim.us@inl.int) ORCID 0000-0001-9875-6188
\end{abstract}

\begin{abstract}
Hexagonal boron nitride (h-BN) has potential applications in protective coatings, single photon emitters and as substrate for graphene electronics. In this paper, we report on the growth of h-BN by chemical vapor deposition (CVD) using ammonia borane as the precursor. Use of CVD allows controlled synthesis over large areas defined by process parameters, e.g. temperature, time, process chamber pressure and gas partial pressures. Furthermore, independently grown graphene and h-BN layers are put together to realize enhancement in electronic properties of graphene.
\end{abstract}

Author Keywords. Hexagonal Boron Nitride, Chemical Vapor Deposition, Ammonia borane, Graphene Electronics

Type: Research Article

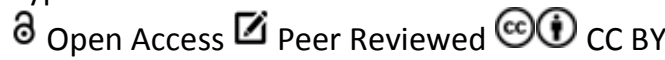

\section{Introduction}

Two dimensional (2D) materials such as graphene and hexagonal boron nitride (h-BN) have attracted scientific and technological interest for potential applications in next generation electronics. The interest is increased since chemical vapor (CVD) synthesis of these 2D materials on large areas was demonstrated (Mattevi, Kim, and Chhowalla 2011). Hexagonal boron nitride ( $\mathrm{h}-\mathrm{BN}$ ) is an electrical insulator that can be regarded as the dielectric equivalent of graphene, in the sense that both materials have $2 \mathrm{D}$ honeycomb lattices that are commensurate (Kim et al. 2012; Xu et al. 2013). In addition to insulating properties, h-BN has high chemical resistance, high thermal stability, low dielectric constant, high mechanical strength and high corrosion resistance leading to potential applications (Meric et al. 2013; Kubota et al. 2007). These properties translate into attractive applications like substrate for graphene electronics, single photon emitters, charge leakage barriers and lubricants (Song et al. 2010). 
Boron nitride (BN) occurs in many allotropic forms, from amorphous thin films (a-BN) analogous to amorphous carbon, to the hexagonal 2D form, which is the most stable of all, and is generally referred to as h-BN. The form analogous to diamond has a cubic lattice, referred to as c-BN, and has thermal and chemical characteristics superior to diamond, though it is much softer than diamond (Wentorf 1957). There is also a rare wurtzite form of boron nitride ( $w-B N$ ) equivalent to carbon lonsdaleite (hexagonal diamond), where crystal structure is more compact, with a hexagonal unit cell rather than a tetragonal one (Silberberg 2009). The $h-B N$ belongs to point group $=D_{6 h}$; space group $=P 6_{3} / \mathrm{mmc}$ having a layered structure with in-plane covalently bonded boron and nitrogen atoms and these layers are held together by weak van der Waal forces. Single crystal basal plane in $\mathrm{h}-\mathrm{BN}$ is not easily broken because of its strong in-plane bonds. Also, in contrast to graphite which displays $A B$ Bernal stacking, h-BN stacks exhibit $A A^{\prime}$ sequences (Kawaguchi, Kuroda, and Muramatsu 2008).

$\mathrm{h}-\mathrm{BN}$ is an excellent platform for graphene electronics. A conventional dielectric such as $\mathrm{SiO}_{2}$ can host impurities, surface roughness and charged surface states, degrading the transport properties of graphene. Whilst h-BN benefits due to atomically smooth surface and free of adsorbed impurities. Dean et al. (2010) investigated the use of h-BN as supporting substrate for graphene which resulted in a 3-4 fold increase of carrier mobility. Several other groups (Wang et al. 2011; Gannett et al. 2011; Meric et al. 2013) reported similar enhancement of graphene transport properties using $\mathrm{h}-\mathrm{BN}$ as a dielectric substrate instead of $\mathrm{SiO}_{2}$. Numerical calculation of thermal conductivity using phonon Boltzmann transport equation is reported as $600 \mathrm{~W} / \mathrm{m} \mathrm{K}$ (Lindsay and Broido 2011). Experimental thermal conductivity on h-BN is reported to be $360 \mathrm{~W} / \mathrm{m} \mathrm{K}$ at room temperature which is many times higher than that of $\mathrm{SiO}_{2}$ making it a better candidate as the dielectric material for heat generating electronics (Jo et al. 2013). All of these studies employed, h-BN flakes exfoliated from h-BN crystals thus limiting technological use of these films. Hence, there is an urgent need to develop an alternative approach to prepare large-scale and high-quality h-BN for present and future applications. CVD presents itself as the most relevant alternative. Moreover, it is already used for the growth of other 2D materials. Yet, precise layer number control and growth conditions are still uncertain. Studies reported in Kim et al. (2012), Ismach et al. (2012) and Lee et al. (2012) does not ascertain precise deposition parameters and sublimating conditions. Here, we present a route for growth of hexagonal boron nitride (h-BN) by CVD using ammonia-borane $\left(\mathrm{H}_{3} \mathrm{~N}-\mathrm{BH}_{3}\right)$ as the $\mathrm{BN}$ precursor with precise details of sublimation conditions.

\section{Materials and methods}

The CVD system (First Nano ET3000) consists of a cylindrical quartz process tube of 5" outer diameter that can accommodate samples measuring up to $10 \mathrm{~cm} \times 15 \mathrm{~cm}$. It has a load lock chamber with transfer arm for loading/unloading samples. Temperature is cascade controlled up to $1200^{\circ} \mathrm{C}$ within the three zone resistively heated furnace. Unlike crude models of CVD where solid precursor is placed inside main process tube, ET3000 has a dedicated remote solid source reservoir connected to the process tube by a heated $1 / 4$ "stainless steel tubing. The sublimating temperatures are applied directly to the reservoir body and top, and can be ramped for desired heating rates.

Ammonia borane ( $\mathrm{AB}$ ) (H3BNH3, also called borazane) is non-flammable and non-explosive under ambient conditions. $A B$ has 1:1 B:N stoichiometry which favors the formation of $h-B N$. $A B$ is chosen instead of borazine, because of its stability in ambient atmosphere whereas Borazine in presence of moisture hydrolyzes to boric acid, ammonia and hydrogen. The method primarily relies on sublimating ammonia borane at $100 \mathrm{C}$ which is then carried into 
the process tube by carrier gas $\mathrm{H}_{2}$. We have grown thin films of $\mathrm{h}-\mathrm{BN}$ on $\mathrm{Cu}$ by sublimating at temperatures from $40{ }^{\circ} \mathrm{C}$ to $100{ }^{\circ} \mathrm{C}$. Growth lasts for one hour at 2 Torr pressure and $1000{ }^{\circ} \mathrm{C}$ temperature. A 4:1 mixture of $\mathrm{Ar}: \mathrm{H}_{2}$ with flow rates of $200: 50 \mathrm{sccm}$ is used as a carrier gas to transport precursor vapor to chamber.

Raman spectra were acquired at room temperature in backscatter geometry using a WiTec Alpha 300 equipped with a $532 \mathrm{~nm}$ frequency-doubled Nd-YAG laser. Data is collected with $600 \mathrm{~g} / \mathrm{mm}$ or $1800 \mathrm{~g} / \mathrm{mm}$ gratings at 50x magnifying objective lens for a laser power level $\sim 10 \mathrm{~mW}$. The $\mathrm{E}_{2 \mathrm{~g}}$ band position is at $\sim 1370 \mathrm{~cm}^{-1}$. Acquisition time was between 10 and $20 \mathrm{~s}$. Raman of $\mathrm{h}-\mathrm{BN}$ on $\mathrm{Cu}$ did not yield any visible signal due to reflection by the Cu background. Hence the Raman spectra measurements were performed on h-BN films transferred onto $\mathrm{Si} / \mathrm{SiO}_{2}$ substrates.

\section{Results and discussions}

The as-grown h-BN layers were characterized by Raman spectroscopy which confirms the $E_{2 g}$ peak in Figure $1 \mathrm{~b}$ at $\sim 1369 \mathrm{~cm}^{-1}$ corresponding to monolayer hexagonal boron nitride. Raman mapping shown in Figure 1 a taken at $(20 \times 20)$ different points in a $(200 \times 200) \mu m^{2}$ area shows a continuous h-BN over whole area. Raman intensity changes at different locations corresponding to thickness variation, but $E_{2 g}$ peak is constant indicating continuous film. In agreement with literature, peak position is thickness dependent, where monolayer ranges from 1367 to $1370 \mathrm{~cm}^{-1}$ and bi layer ranges from 1363 to $1365 \mathrm{~cm}^{-1}$ (Gorbachev et al. 2011).

a)
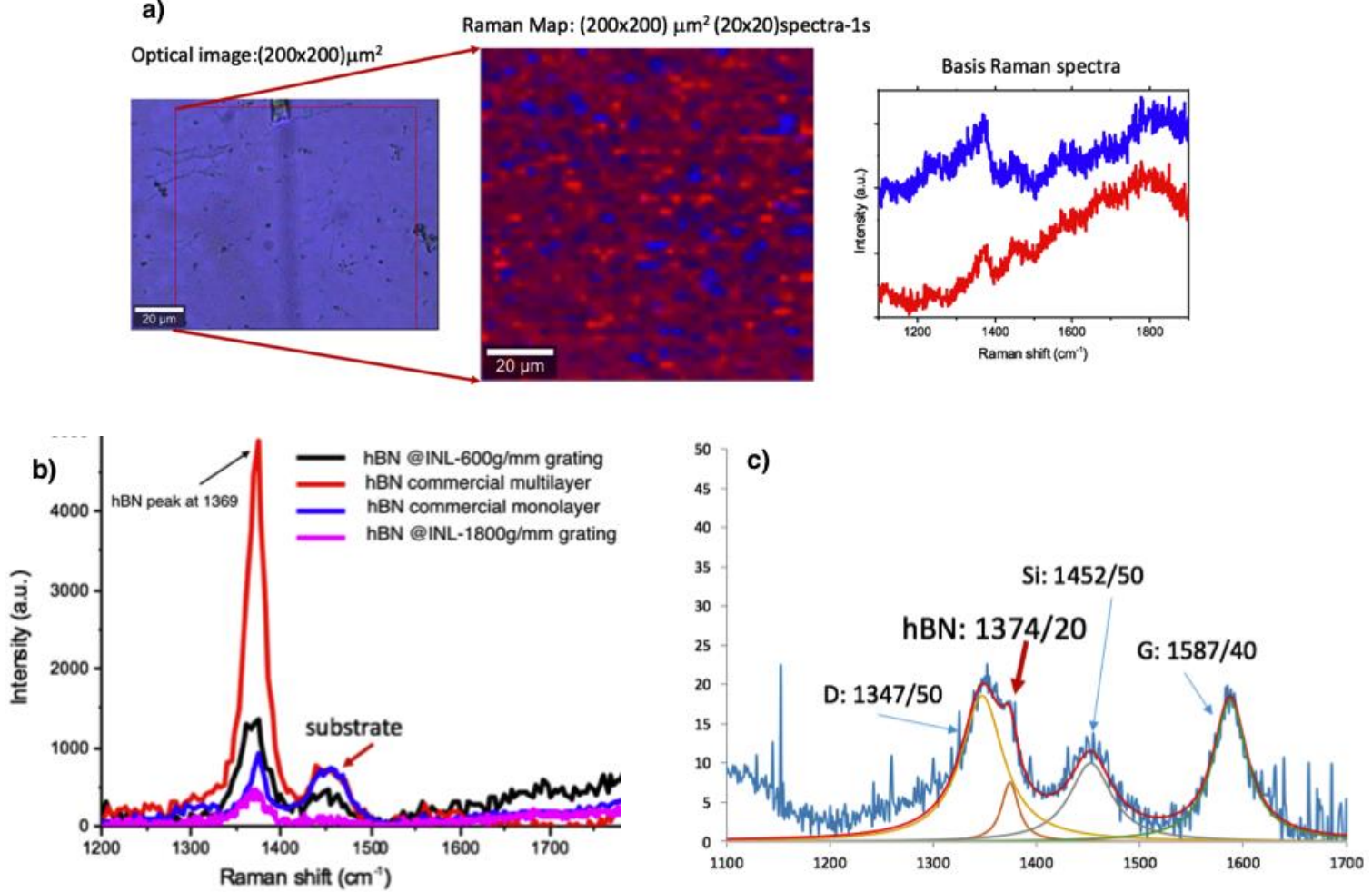

Figure 1: a) h-BN coverage post transfer to $\mathrm{Si} / \mathrm{SiO}_{2}$ b) Raman peak at $\sim 1369 \mathrm{~cm}^{-1}$ identifying monolayer $\mathrm{h}$ BN c) Raman of graphene / h-BN stacked layer showing peak assignment: position/FWHM

AFM images were collected using Bruker dimension icon in tapping mode with tapping frequency of $\sim 100 \mathrm{KHz}$ and a scan rate of $0.5 \mathrm{~Hz}$ with sampling of $256 \times 256$ pixels and scan area of 40 square microns. Images were analyzed using Image SXM 200 software. It is clear that surface corrugations are much large on $\mathrm{SiO}_{2}$ compared to $\mathrm{h}-\mathrm{BN}$. This agrees with the planar nature of h-BN as compared to amorphous $\mathrm{SiO}_{2}$. Root mean square roughness measured for bare $\mathrm{SiO}_{2}$ is $11.3 \pm 0.5 \mathrm{~nm}$ and for $\mathrm{h}-\mathrm{BN}$ on $\mathrm{SiO}_{2}$ is $6.1 \pm 0.5 \mathrm{~nm}$. As shown in Figure 2, hexagonal 
boron nitride is atomically very smooth when compared to $\mathrm{SiO}_{2}$. Results are in agreement with previously reported values (Xue et al. 2011). Thickness of monolayer measured using AFM scan and was found to be $0.4 \mathrm{~nm}$. This is closely in agreement with thickness observation of $0.33 \mathrm{~nm}$ in literature (Golla et al. 2013).
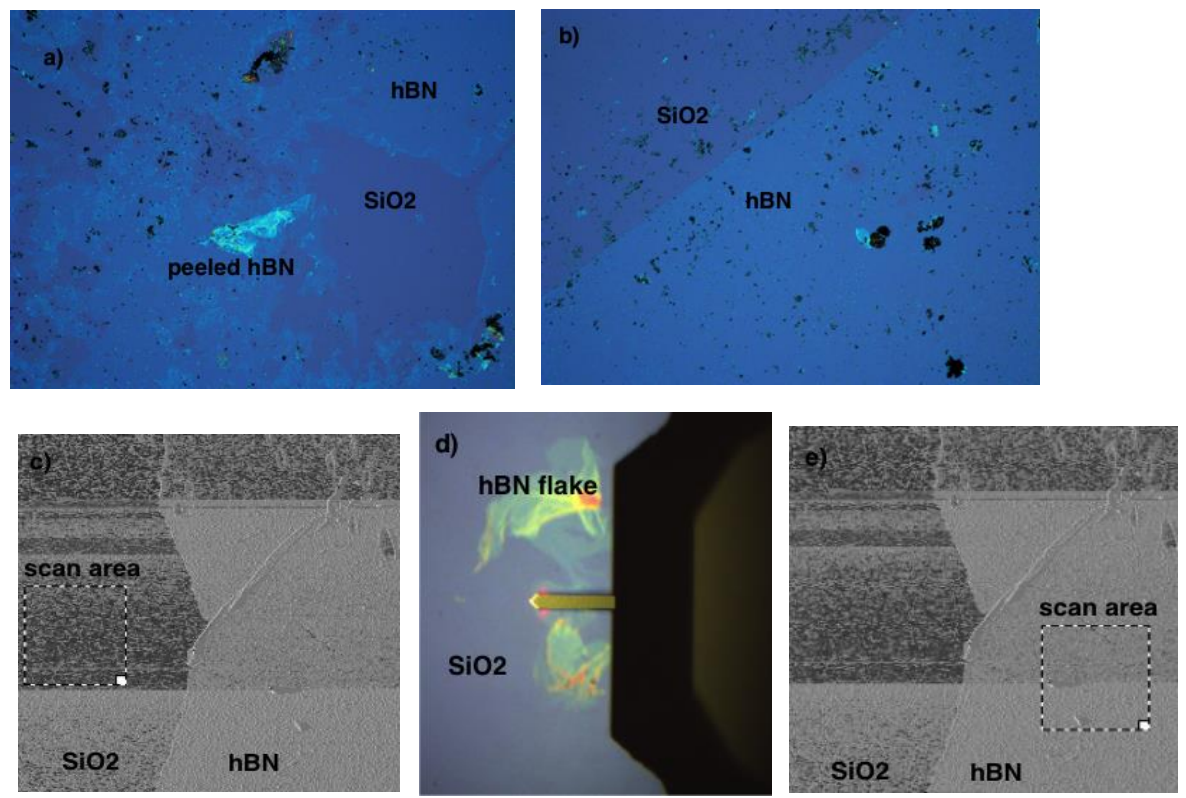

Figure 2: a) Optical image of h-BN peeled off on $\mathrm{Si} / \mathrm{SiO} 2 \mathrm{~b}$ ) Optical image of hBN on $\mathrm{Si} / \mathrm{SiO} 2$ edge c,e) AFM roughness scan of $\mathrm{hBN}$ and $\mathrm{Si} / \mathrm{SiO} 2$

\subsection{Growth of $h-B N$ on copper}

It has been reported that $\mathrm{h}-\mathrm{BN}$ monolayers readily grow with hexagonal structure of transition metal catalysts such as, $\mathrm{Cu}, \mathrm{Ni}, \mathrm{Pt}$, in (111) direction. Preobrajenski, Vinogradov, and Mårtensson (2005) showed that h-BN film on Cu (111) has a commensurate structure, which is stable when $\mathrm{N}$ atoms are bound directly over $\mathrm{Cu}$ atoms and $\mathrm{B}$ atoms over $\mathrm{FCC}$ hollow sites resulting in a film under slightly compressive stress. As shown in the Table 1, Laskowski, Blaha, and Schwarz (2008) reported binding energies of h-BN on different transition metals through density functional theory (DFT) calculations. Metals with high binding energy and large lattice mismatches, greater than $10 \%$, like Pt and Ir, can cause Nano mesh-like film structure due to strong straining of $h-B N \sigma$ bonds (Preobrajenski, Vinogradov, and Mårtensson 2005).

High binding energy with good lattice match to the metal catalyzer should be considered when choosing a suitable substrate for $\mathrm{h}-\mathrm{BN}$ deposition. Ni and Cu having a lattice mismatch of $0.4 \%$ and $0.9 \%$, respectively, are considered ideal for synthesizing commensurate $\mathrm{h}-\mathrm{BN}$ layers (Joshi et al. 2012). h-BN layers on Ni foils are considered leakier as dielectric layer due to incomplete regions present at $\mathrm{Ni}$ grain boundaries (Bresnehan et al. 2014). Hence, growth on Cu foils is realistic and suitable for upscaling. For dielectric applications the tunneling currents were found to be excessive for h-BN films less than four layers thick (Britnell et al. 2012).

\begin{tabular}{|c|c|}
\hline Element & $\Delta \mathrm{E}$ (Binding energy by LDA) \\
\hline $\mathrm{Co}$ & 0.32 \\
\hline $\mathrm{Ni}$ & 0.27 \\
\hline $\mathrm{Cu}$ & 0.19 \\
\hline $\mathrm{Ir}$ & 0.49 \\
\hline $\mathrm{Pt}$ & 0.34 \\
\hline $\mathrm{Au}$ & 0.16 \\
\hline $\mathrm{Ag}$ & 0.19 \\
\hline
\end{tabular}

Table 1: Binding energies of $\mathrm{h}-\mathrm{BN}$ on different transition metals. Reprinted table with permission with Laskowski, Blaha, and Schwarz (2008) 


\subsection{Sublimation of $A B$}

The results show enhanced dehydrogenation of $A B$ at $80^{\circ} \mathrm{C}$ only after it was pre-heated at the same temperature for four hours. Thermal treatments of $A B$ were performed in the same reservoir with dummy runs. With increased pre heating duration the dehydrogenation is faster and effective. This was also realized by the group of (Zhang et al. 2010) for the study of $A B$ as solid source of hydrogen fuel. During the preheating stage the solid $A B$ is decomposed to diammoniate of diborane (DADB, $\left.\left[\left(\mathrm{NH}_{3}\right)_{2} \mathrm{BH}_{2}\right]^{+}\left[\mathrm{BH}_{4}\right]^{-}\right)$and few other polymeric and oligomeric species of $\mathrm{BH}_{2} \mathrm{~N}_{2}$. This reaction shifts the melting point of $A B$ to a lower value (Stowe et al. 2007). After the pre-heating stage h-BN growth is rapid. An understanding of this incubation period of $A B$ allows for a significant reduction in $\mathrm{h}-\mathrm{BN}$ growth time and a greater control over the process.

At low sublimating temperatures, the ammonia borane precursors are not fully evaporated. Series of runs with every $5{ }^{\circ} \mathrm{C}$ raise in temperature were performed. High quality films with good substrate coverage were achieved at sublimating temperatures of $100{ }^{\circ} \mathrm{C}$. Thermal decomposition of $A B$ takes place in several weight loss steps releasing $\mathrm{H}_{2}$ and $B N$ complexes. Starting with an endothermic process at $101{ }^{\circ} \mathrm{C}$, resulting in the melting of $A B$, followed by exothermic decomposition steps at $\sim 110, \sim 130$ and $1170{ }^{\circ} \mathrm{C}$. The first weight loss at $110{ }^{\circ} \mathrm{C}$ releases $\mathrm{H}_{2}$ and monomeric amino borane $\left(\mathrm{BH}_{2} \mathrm{NH}_{2}\right)$ with residual solid polyaminoborane. Second weight loss step at $130{ }^{\circ} \mathrm{C}$ results in further hydrogen release along with borazine $\left(\mathrm{B}_{3} \mathrm{~N}_{3} \mathrm{H}_{6}\right)$. Finally, at $1170^{\circ} \mathrm{C}$ hydrogen is fully released, resulting in the formation of crystalline hexagonal boron nitride phase (Frueh et al. 2011).

\subsection{Graphene/h-BN hetero-structure}

Graphene was transferred onto $\mathrm{h}-\mathrm{BN} / \mathrm{Si} / \mathrm{SiO} 2$ substrates using $\mathrm{Cu}$ etch method. For that, the as-grown $\mathrm{h}$-BN films have to be first decoupled from the native $\mathrm{Cu}$ substrate and then transferred onto $\mathrm{Si} / \mathrm{SiO}_{2}$ wafers, using a temporary, polymeric substrate (poly-methylmethacrylate, PMMA). Post transfer the samples were annealed for 12 hours at $150{ }^{\circ} \mathrm{C}$ and the process is repeated to transfer graphene. The resulting Graphene/h-BN heterostructure was measured for Raman signature. The Raman spectrum shown in Figure 1c shows prominent G-line centered at $1587 \mathrm{~cm}^{-1}$ and Full-width at half maximum (FWHM) $49 \mathrm{~cm}^{-1}$, as expected for graphene. The peak centered at $1374 \mathrm{~cm}^{-1}\left(\mathrm{FWHM}=20 \mathrm{~cm}^{-1}\right.$ ) can be attributed to Raman active LO-phonon in h-BN. It is crucial to distinguish this peak from defect induced D-line in graphene, appearing around $1347 \mathrm{~cm}^{-1}\left(F W H M=50 \mathrm{~cm}^{-1}\right)$. Study by optical microscopy shows presence of impurities accumulated during the transfer. It is challenging to prevent adsorption of water molecules and other chemicals at the interface during the transfer. Due to these fundamental challenges graphene grown over h-BN film will exhibit better performance as the interface is protected from external impurities. Extensive characterization revealed a stable heterostructure which can be further patterned to make devices, e.g. a field effect transistor.

\section{Conclusions}

In this work, we successfully synthesized large area h-BN films using low pressure CVD. The results shown above support past observations in the literature and detail additional mechanistic insight into the elementary process that lead to pristine $h-B N$ films by thermal decomposition of solid AB. The layers were characterized using Optical microscopy, Raman spectroscopy and AFM. The thickness of monolayer was found to be $0.4 \mathrm{~nm}$. 


\section{References}

Bresnehan, Michael S., Ganesh R. Bhimanapati, Ke Wang, David W. Snyder and Joshua A. Robinson. 2014. "Impact of copper overpressure on the synthesis of hexagonal boron nitride atomic layers". ACS Applied Materials \& Interfaces 6 (19):16755-16762. Accessed August 29, 2017. DOI: 10.1021/am503844u.

Britnell, L., R. V. Gorbachev, R. Jalil, B. D. Belle, F. Schedin, M. I. Katsnelson, L. Eaves, S. V. Morozov, A. S. Mayorov, N. M. R. Peres, A. H. C. Neto, J. Leist, A. K. Geim, L. A. Ponomarenko and K. S. Novoselov. 2012. "Electron tunneling through ultrathin boron nitride crystalline barriers". Nano Letters 12 (3):1707-1710. Accessed August 29, 2017. DOI: $10.1021 / \mathrm{nl} 3002205$.

Dean, C. R., A. F. Young, I. Meric, C. Lee, L. Wang, S. Sorgenfrei, K. Watanabe, T. Taniguchi, P. Kim, K. L. Shepard and J. Hone. 2010. "Boron nitride substrates for high-quality graphene electronics". Nature Nanotechnology 5 (10):722-726. Accessed August 29, 2017. DOI: 10.1038/nnano.2010.172.

Frueh, S., R. Kellett, C. Mallery, T. Molter, W. S. Willis, C. King'ondu and S. L. Suib. 2011. "Pyrolytic decomposition of ammonia borane to boron nitride". Inorganic Chemistry 50 (3):783-792. Accessed August 29, 2017. DOI: 10.1021/ic101020k.

Gannett, W., W. Regan, K. Watanabe, T. Taniguchi, M. F. Crommie and A. Zettl. 2011. "Boron nitride substrates for high mobility chemical vapor deposited graphene". Applied Physics Letters 98 (24). Accessed August 29, 2017. DOI: 10.1063/1.3599708.

Golla, D., K. Chattrakun, K. Watanabe, T. Taniguchi, B. J. LeRoy and A. Sandhu. 2013. "Optical thickness determination of hexagonal boron nitride flakes". Applied Physics Letters 102 (16). Accessed August 29, 2017. DOI: 10.1063/1.4803041.

Gorbachev, R. V., I. Riaz, R. R. Nair, R. Jalil, L. Britnell, B. D. Belle, E. W. Hill, K. S. Novoselov, K. Watanabe, T. Taniguchi, A. K. Geim and P. Blake. 2011. "Hunting for monolayer boron nitride: Optical and raman signatures". Small 7 (4):465-468. Accessed August 29, 2017. DOI: 10.1002/smll.201001628.

Ismach, A., H. Chou, D. A. Ferrer, Y. P. Wu, S. McDonnell, H. C. Floresca, A. Covacevich, C. Pope, R. Piner, M. J. Kim, R. M. Wallace, L. Colombo and R. S. Ruoff. 2012. "Toward the controlled synthesis of hexagonal boron nitride films". ACS Nano 6 (7):6378-6385. Accessed August 29, 2017. DOI: 10.1021/nn301940k.

Jo, I., M. T. Pettes, J. Kim, K. Watanabe, T. Taniguchi, Z. Yao and L. Shi. 2013. "Thermal conductivity and phonon transport in suspended few-layer hexagonal boron nitride". Nano Letters 13 (2):550-554. Accessed August 29, 2017. DOI: 10.1021/nl304060g.

Joshi, S., D. Ecija, R. Koitz, M. Iannuzzi, A. P. Seitsonen, J. Hutter, H. Sachdev, S. Vijayaraghavan, F. Bischoff, K. Seufert, J. V. Barth and W. Auwarter. 2012. "Boron nitride on Cu(111): An electronically corrugated monolayer". Nano Letters 12 (11):5821-5828. Accessed August 29, 2017. DOI: 10.1021/nl303170m.

Kawaguchi, M., S. Kuroda and Y. Muramatsu. 2008. "Electronic structure and intercalation chemistry of graphite-like layered material with a composition of BC6N". Journal of Physics and Chemistry of Solids 69 (5-6):1171-1178. Accessed August 29, 2017. DOI: 10.1016/j.jpcs.2007.10.076.

Kim, K. K., A. Hsu, X. T. Jia, S. M. Kim, Y. S. Shi, M. Hofmann, D. Nezich, J. F. Rodriguez-Nieva, M. Dresselhaus, T. Palacios and J. Kong. 2012. "Synthesis of monolayer hexagonal boron nitride on $\mathrm{Cu}$ foil using chemical vapor deposition". Nano Letters 12 (1):161-166. 
Accessed August 29, 2017. DOI: 10.1021/nI203249a.

Kubota, Y., K. Watanabe, O. Tsuda and T. Taniguchi. 2007. "Deep ultraviolet light-emitting hexagonal boron nitride synthesized at atmospheric pressure". Science 317 (5840):932934. Accessed August 29, 2017. DOI: 10.1126/science.1144216.

Laskowski, R., P. Blaha and K. Schwarz. 2008. "Bonding of hexagonal BN to transition metal surfaces: An ab initio density-functional theory study". Physical Review $B 78$ (4). Accessed August 29, 2017. DOI: 10.1103/PhysRevB.78.045409.

Lee, K. H., H. J. Shin, J. Lee, I. Y. Lee, G. H. Kim, J. Y. Choi and S. W. Kim. 2012. "Large-scale synthesis of high-quality hexagonal boron nitride nanosheets for large-area graphene electronics". Nano Letters 12 (2):714-718. Accessed August 29, 2017. DOI: $10.1021 / \mathrm{nl} 203635 \mathrm{v}$.

Lindsay, L. and D. A. Broido. 2011. "Enhanced thermal conductivity and isotope effect in singlelayer hexagonal boron nitride". Physical Review B 84 (15). Accessed August 29, 2017. DOI: 10.1103/PhysRevB.84.155421.

Mattevi, C., H. Kim and M. Chhowalla. 2011. "A review of chemical vapour deposition of graphene on copper". Journal of Materials Chemistry 21 (10):3324-3334. Accessed August 29, 2017. DOI: 10.1039/c0jm02126a.

Meric, I., C. R. Dean, N. Petrone, L. Wang, J. Hone, P. Kim and K. L. Shepard. 2013. "Graphene field-effect transistors based on boron-nitride dielectrics". Proceedings of the IEEE 101 (7):1609-1619. Accessed August 29, 2017. DOI: 10.1109/Jproc.2013.2257634.

Preobrajenski, A. B., A. S. Vinogradov and N. Martensson. 2005. "Monolayer of h-BN chemisorbed on $\mathrm{Cu}(111)$ and $\mathrm{Ni}(111)$ : The role of the transition metal 3d states". Surface Science 582 (1-3):21-30. Accessed August 29, 2017. DOI: 10.1016/j.susc.2005.02.047.

Silberberg, Martin S. 2009. Chemistry the molecular nature of matter and change. Boston: McGraw-Hill Higher Education.

Song, L., L. J. Ci, H. Lu, P. B. Sorokin, C. H. Jin, J. Ni, A. G. Kvashnin, D. G. Kvashnin, J. Lou, B. I. Yakobson and P. M. Ajayan. 2010. "Large scale growth and characterization of atomic hexagonal boron nitride layers". Nano Letters 10 (8):3209-3215. Accessed August 29, 2017. DOI: $10.1021 / \mathrm{nl} 1022139$.

Stowe, A. C., W. J. Shaw, J. C. Linehan, B. Schmid and T. Autrey. 2007. "In situ solid state B-11 MAS-NMR studies of the thermal decomposition of ammonia borane: mechanistic studies of the hydrogen release pathways from a solid state hydrogen storage material". Physical Chemistry Chemical Physics 9 (15):1831-1836. Accessed August 29, 2017. DOI: 10.1039/b617781f.

Wang, H., T. Taychatanapat, A. Hsu, K. Watanabe, T. Taniguchi, P. Jarillo-Herrero and T. Palacios. 2011. "BN/Graphene/BN Transistors for RF applications". IEEE Electron Device Letters 32 (9):1209-1211. Accessed August 29, 2017. DOI: 10.1109/Led.2011.2160611.

Wentorf, R. H. 1957. "Cubic form of boron nitride". Journal of Chemical Physics 26 (4):956956. Accessed August 29, 2017. DOI: 10.1063/1.1745964.

Xu, M. S., T. Liang, M. M. Shi and H. Z. Chen. 2013. "Graphene-like two-dimensional materials". Chemical Reviews 113 (5):3766-3798. Accessed August 29, 2017. DOI: 10.1021/cr300263a.

Xue, J. M., J. Sanchez-Yamagishi, D. Bulmash, P. Jacquod, A. Deshpande, K. Watanabe, T. Taniguchi, P. Jarillo-Herrero and B. J. Leroy. 2011. "Scanning tunnelling microscopy and spectroscopy of ultra-flat graphene on hexagonal boron nitride". Nature Materials 10 (4):282-285. Accessed August 29, 2017. DOI: 10.1038/nmat2968. 
Zhang, J. S., Y. Zhao, D. L. Akins and J. W. Lee. 2010. "Thermal decomposition and spectroscopic studies of preheated ammonia borane". Journal of Physical Chemistry C 114 (45):1952919534. Accessed August 29, 2017. DOI: 10.1021/jp105014t. 